\title{
Flame evolution in shock-accelerated flow under different reactive gas mixture gradients
}

\author{
Yuejin Zhu, ${ }^{1,2}$ Longkun Gao, ${ }^{1}$ Kai Hong Luo, ${ }^{2, *}$ Jianfeng Pan,,${ }^{1, \dagger}$ Zhenhua Pan, ${ }^{1}$ and Penggang Zhang ${ }^{1}$ \\ ${ }^{1}$ School of Energy and Power Engineering, Jiangsu University, Zhenjiang 212013, China \\ ${ }^{2}$ Department of Mechanical Engineering, University College London, Torrington Place, London WC1E 7JE, United Kingdom
}

(Received 19 February 2019; published 23 July 2019)

\begin{abstract}
The interaction between a planar shock wave and a spherical flame is studied numerically for an ethyleneoxygen-nitrogen gas mixture. Influences of different initial reactive gas mixture gradients on the shock-flame interaction are investigated by using high-resolution computational simulations. The results show that the different reactive gas mixture gradients can greatly affect the flame evolution in shock accelerated flow. A detonation only emerges in the homogenous reactive gas mixture case, but a distinct shock bifurcation can be found in the inhomogeneous cases where the leftward reflected shock wave propagates in a reverse flow with a high transverse velocity gradient in the inhomogeneous cases. Also, the flame volume and heat release rate increase when the distribution of the reactive gas mixture is uniform or with a positive gradient in this paper, but decrease when the distribution of the reactive gas mixture is with a negative gradient, however, the ratio of unburned to burned regions in the flame zone shows just the opposite trends. Furthermore, the factors affecting the vorticity generation are also analyzed. It is found that the compression term has a relatively stronger influence on the vorticity generation in all the three cases except the period before the reflected shock wave impinges on the distorted flame in the homogeneous case, wherein the baroclinic effect dominates the vorticity generation in the flame zone.
\end{abstract}

DOI: 10.1103/PhysRevE.100.013111

\section{INTRODUCTION}

Flames in the reactive gas mixture subjected to the shock compression are usually unstable. This phenomenon is mainly due to the Richtmyer-Meshkov (RM) instability, and frequently occurs in industrial applications, such as the supersonic combustion propulsion [1,2] and industrial explosions [3]. It involves many complicated physics and chemical processes, which have not been fully understood.

A typical configuration to study the flame instability in shock accelerated flow is the shock-flame interaction, which has been widely investigated experimentally and numerically. Thomas et al. [4] experimentally studied the shock-flame interaction, and found that the RM instability was the main mechanism for the flame instability. Oran et al. [3,5,6] carried out a series of numerical computations on the shock-flame interaction, and found there would emerge hot spots and even detonation under a certain condition. They also found that the flame could be greatly disturbed and accelerated when a stronger reflected shock wave from the right end wall impinged on the distorted flame again. In this case, a complex reactive shock bifurcation structure (also called "strange wave" structure) may emerge via the shock wave-flame-boundary layer interactions. During the same period, Teng et al. [7] numerically investigated the interaction between shock wave and deflagration flame, and the results revealed that a detonation emerged via RM instability. In recent years, Taylor et al. [8] conducted numerical research on the process when a planar

\footnotetext{
*k.luo@ucl.ac.uk

†mike@ujs.edu.cn
}

shock wave impinged on a circle laminar flame, and two different detonation initiation mechanisms were proposed: direct ignition by reflected shock wave and a gradient mechanism involving hot spots. Moreover, Zhu et al. $[9,10]$ numerically studied the shock-flame interaction by using a third-order accurate five-point total variation diminishing scheme, and the influences of initial parameters (flame size and number, shock wave strength, and gas mixture reactivity) on the flame evolution were investigated in detail. It was found that the morphology of the flame evolution showed the severe expansion and corrugation disturbed by shock waves, especially by reflected shock wave. The results also showed that the detonation might emerge in the flow fields under a certain condition, but no further studies were made on the evolution of the detonation wave.

Despite the extensive experimental and numerical studies that have been made in the past few decades, many issues concerning the shock-flame interaction are still not been resolved. Especially for the practical situations, inhomogeneity in the reactive gas mixture will have a significant influence on the flame evolution. Actually, at present, the influence of inhomogeneity of the reactive gas mixture on the combustion processes has aroused widespread interest [11-14], but few studies have been devoted to the shock-flame interaction topic. The investigation of the shock-flame interaction in the inhomogeneous reactive gas mixture will help us gain more insight, particularly when a high intensity shock wave is adopted. Hence, in this study, a highly accurate numerical scheme is employed to study the spherical flame evolution in the strong shock accelerated flow. The evolution of shock wave and distorted flame is analyzed in detail, and the timedependent integral properties are also clarified. The main aim 
is to investigate the influence of different reactive gas mixture gradients on the shock-flame interaction process.

\section{NUMERICAL METHOD AND SETUP}

\section{A. Numerical method}

The two-dimensional axisymmetrical reactive NavierStokes (N-S) equations coupled with one-step Arrhenius chemical reaction are utilized to simulate the shock-flame interaction, expressed as follows:

$$
\frac{\partial U}{\partial t}+\frac{\partial F}{\partial x}+\frac{\partial G}{\partial y}+W=Q
$$

where

$$
\begin{aligned}
& U=\left[\begin{array}{l}
\rho \\
\rho u \\
\rho v \\
E \\
\rho Y
\end{array}\right], \quad F=\left[\begin{array}{c}
\rho u \\
\rho u^{2}+p-\tau_{x x} \\
\rho u v-\tau_{y x} \\
(E+p) u-k \partial T / \partial x-u \tau_{x x}-v \tau_{y x} \\
\rho u Y-D \rho \partial Y / \partial x
\end{array}\right], \\
& G=\left[\begin{array}{c}
\rho v \\
\rho u v-\tau_{x y} \\
\rho v^{2}+p-\tau_{y y} \\
(E+p) v-k \partial T / \partial y-u \tau_{x y}-v \tau_{y y} \\
\rho v Y-D \rho \partial Y / \partial y
\end{array}\right], \\
& W=\frac{v}{y}\left[\begin{array}{c}
\rho \\
\rho u \\
\rho v \\
p+E \\
\rho Y
\end{array}\right]
\end{aligned}
$$

$\rho$ is the density, $u$ and $v$ are the velocities in the $x$ and $y$ directions, respectively. $E$ is the total energy which can be expressed as $E=p /(\gamma-1)+0.5 \rho \sum_{i=1}^{2} u_{i}^{2}+\rho q S, p$ is the pressure, $S$ is the mass fraction of the ambient fresh reactive gas (the combustible mixture of fuel and air for a premixed reactive system). $\tau_{x x}, \tau_{x y}, \tau_{y x}$, and $\tau_{y y}$ are the viscous stresses, and can be expressed as $\tau_{x x}=\rho v(4 / 3(\partial u / \partial x)-$ $2 / 3(\partial v / \partial y)), \quad \tau_{x y}=\tau_{y x}=\rho v(\partial u / \partial x+\partial v / \partial y)$, and $\tau_{y y}=$ $\rho v(4 / 3(\partial v / \partial y)-2 / 3(\partial u / \partial x))$. The kinematic viscosity $v$, diffusion $D$, and heat conduction $k$ have a similar exponential dependence on temperature [3,9]:

$$
v=v_{0} \frac{T^{n}}{\rho}, \quad D=D_{0} \frac{T^{n}}{\rho}, \quad \frac{k}{\rho c_{p}}=k_{0} \frac{T^{n}}{\rho},
$$

where $v_{0}, D_{0}$, and $k_{0}$ are constants, $T$ is the temperature, $c_{p}$ is the specific heat capacity at constant pressure, and $n=0.7$. In addition, $W$ denotes the axisymmetric correction term, $Q$ is the source term, $\dot{\omega}$ is the chemical reaction speed and can be written as $\dot{\omega}=A \rho S \exp \left(-E_{a} /(R T)\right)$, where $A$ is the preexponential factor, $E_{a}$ is the activation energy and $R$ is the universal gas constant $[3,9,10]$.

To solve the N-S equations, the splitting algorithm is applied. A global Lax-Friedrichs flux vector splitting combined with a ninth-order weighted essentially nonoscillatory scheme [15] is adopted to discretize the spatial derivatives of inviscid fluxes. A tenth-order central difference scheme is employed for the viscous fluxes. For the time advancement, a third-order

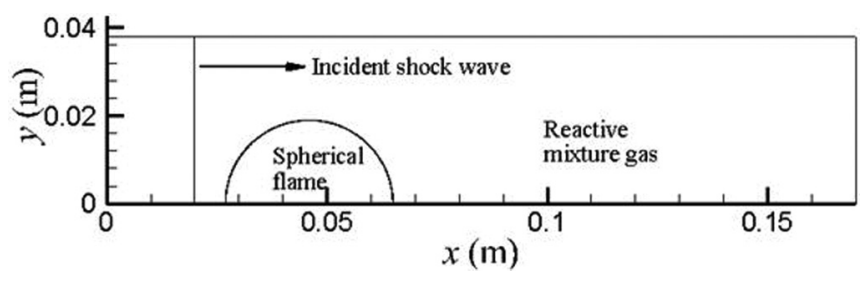

FIG. 1. Schematic of the computational setup.

Runge-Kutta algorithm is used. The present numerical method has been utilized successfully in previous studies [16-18].

\section{B. Computational setup}

The computational setup is shown in Fig. 1. The spherical flame with density of $0.01578 \mathrm{~kg} / \mathrm{m}^{3}$ is surrounded by a fresh reactive gas mixture $\mathrm{C}_{2} \mathrm{H}_{4} / 3 \mathrm{O}_{2} / 4 \mathrm{~N}_{2}$ with initial conditions of $r_{0}=0.1615 \mathrm{~kg} / \mathrm{m}^{3}, T_{0}=293 \mathrm{~K}$ at $p_{0}=13.3 \mathrm{kPa}$, which is the same as the one chosen by a previous experimental study [4]. The incident shock wave initially locates at $x=0.02 \mathrm{~m}$, and then moves from left to right to impinge on the spherical flame, and when it reflects from the right end wall, the reflected shock wave will impinge on the distorted flame again.

In this computations, the inflow condition is enforced on the surface of $x=0 \mathrm{~m}$, whose values of the flow variables are the same as those behind the incident shock wave. Besides, the adiabatic and no-slip wall condition is enforced on the other surfaces of computational domain. In order to simplify the calculation, an axisymmetrical boundary condition is utilized at the bottom $(y=0 \mathrm{~m})$ of the computational domain. The adopted uniform grid size in this paper is $\Delta x=\Delta y=$ $0.1 \mathrm{~mm}$, which can fully and correctly describe the flame development [18]. Also, it is assumed that the Lewis number is unity, so the constants are set to $u_{0}=D_{0}=k_{0}=3.2 \times$ $10^{-7} \mathrm{~kg} /\left(\mathrm{s} \mathrm{m} \mathrm{K}^{0.7}\right)[9,10,18]$. For the gas mixture adopted in this paper, the $E_{a} / R T_{0}$ is set to 38.2 and the value of $A=$ $1.2 \times 10^{8} \mathrm{~m}^{3} /(\mathrm{kg} \mathrm{s})$ according to our previous studies $[9,10]$.

\section{RESULTS AND DISCUSSION}

\section{A. Numerical validation}

The numerical results are first compared with the experiments of Thomas et al. [4]. Figure 2 gives the comparison between experimental Schlieren images [4] and computational Schlieren results at the selected time instants. Figures 2(a) and 2(b) show the scenarios of incident shock wave (Mach number is 1.7) and spherical flame interaction. It can be found that the spherical flame distorts via RM instability behind the incident shock wave, and forms a pair of longitudinal symmetrical flame. Moreover, Figs. 2(c) and 2(d) correspond to the interaction of reflected shock wave with the distorted flame, and the flame expands drastically. It should be noted that the experimental Schlieren result represents the threedimensional projection of light, but the numerical result is obtained from two-dimensional simulation, thus the distorted flame in the experimental result seems larger than that in the numerical result. This result presents similarities with those reported by Dong et al. [19]. In short, good qualitative agreement in the distorted flame and shock wave evolution 

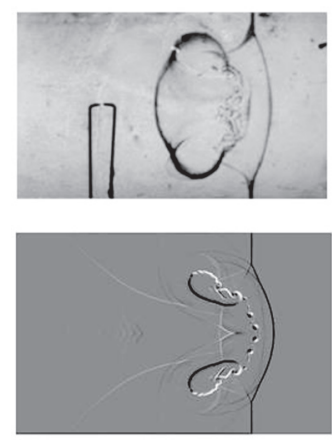

(a)
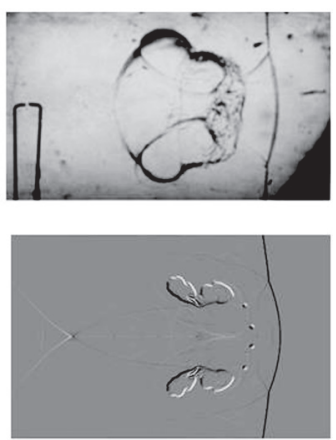

(b)
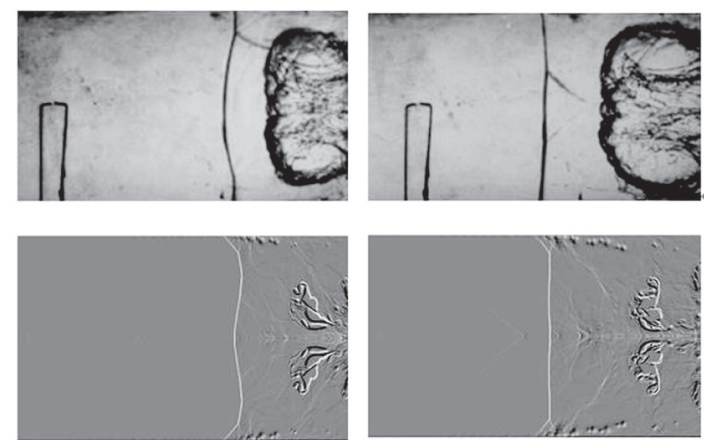

(c)

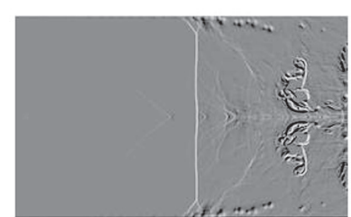

(d)

FIG. 2. Comparison between experimental Schlieren images [4] and computational Schlieren images at the selected time instants (a) $t=143 \mu \mathrm{s}$, (b) $t=194 \mu \mathrm{s}$, (c) $t=443 \mu \mathrm{s}$, and (d) $t=494 \mu \mathrm{s}$.

between the numerical simulation and experiment is obtained, which indicates the reliability of the adopted numerical approach.

\section{B. Visualized flow fields}

In this study, three cases with different initial reactive gas mixture gradients for the shock-flame interaction are investigated, with the same high initial shock Mach number $(\mathrm{Ma}=$ 2.51). Specifically, the three typical and simple different fresh reactive gas mixture distributions are defined as follows:

$$
S= \begin{cases}1, & \text { case } 1 \\ \frac{1+\left(2 y / L_{y}\right)^{2}}{5}, & \text { case 2 } \\ \frac{1}{1+\left(2 y / L_{y}\right)^{2}}, & \text { case 3 }\end{cases}
$$

where $L_{y}$ is the height of the computational domain in the $y$ direction. The different distributions of the fresh reactive gas mixture in the three cases are shown in Fig. 3. It can be found that case 1 corresponds to the uniform distribution of the reactive gas mixture, the distribution in case 2 has a positive gradient, while the distribution in case 3 has a negative gradient.

Figure 4 presents the evolution of flame and waves in the incident and reflected shock accelerated flow in case 1, where the top half of each figure is the density distribution

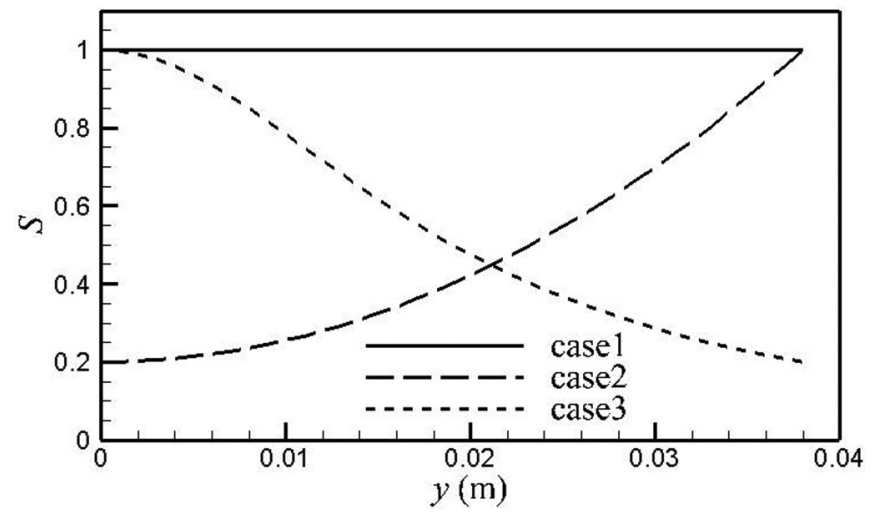

FIG. 3. Reactive gas mixture distributions in the three different cases. while the bottom half is the reactive gas mass distribution. Figures 4(a) and 4(b) correspond to the time instants when the incident shock wave has passed through the initial spherical flame. The acoustic impedance in the flame zone is smaller than that outside because the density of flame is smaller than the ambient fresh reactive gas, thus the shock wave in the flame zone moves faster than the outside in Figs. 4(a) and 4(b). Meanwhile, the initial flame forms a pair of longitudinal symmetrical flames. When the reflected shock wave passes through the distorted flame in Fig. 4(c), the curving reflected shock wave collides in the vicinity of the symmetrical axis of the flow field to form a local high pressure zone. Then, the local hot spot in this high pressure zone will develop into the detonation owing to the surrounding abundant fresh reactive gas mixture and high temperature in Fig. 4(d). By checking the numerical result, the propagation velocity of the detonation wave is about $1997 \mathrm{~m} / \mathrm{s}$ and the pressure behind the detonation wave is about $1.1 \mathrm{MPa}$, which are both close to the theoretical Chapman-Jouguet detonation values (2041 $\mathrm{m} / \mathrm{s}$ and $1.17 \mathrm{MPa}$, obtained by the calculation of the GordonMcbride code [20]). The detonation wave expands quickly and the fresh reactive gas mixture is also consumed fast in Fig. 4(e). At $t=231.2 \mu \mathrm{s}$, the expanding detonation wave reflects from the top wall to form a new downward reflected shock wave RSW1 and a leftward Mach stem. Subsequently, the curved downward RSW1 collides in the vicinity of the symmetrical axis of the flow field again to form a new local high pressure zone and a new upward shock wave RSW2 in Figs. 4(g) and 4(h). Hence, a detonation emerges behind the leftward reflected shock wave due to the local high pressure zone in the vicinity of the symmetrical axis of the flow field. Subsequently, the expanding detonation wave will turn into a planar detonation wave with many triple points and transverse waves. To some extent, the revelation of the complicated waves in this case indicates the superiority of this high-order numerical method in this study.

For case 2 with the positive reactive gas mixture gradient, Fig. 5 gives the evolution of flame and waves in the reflected shock accelerated flow. A local high pressure zone is formed in Fig. 5(a) owing to the same reason as that in Fig. 4(c). Then, the surrounding waves of this local high pressure zone form an upward shock wave RSW1 and a leftward shock wave RSW2 in Figs. 5(b) and 5(c). Moreover, it should be 


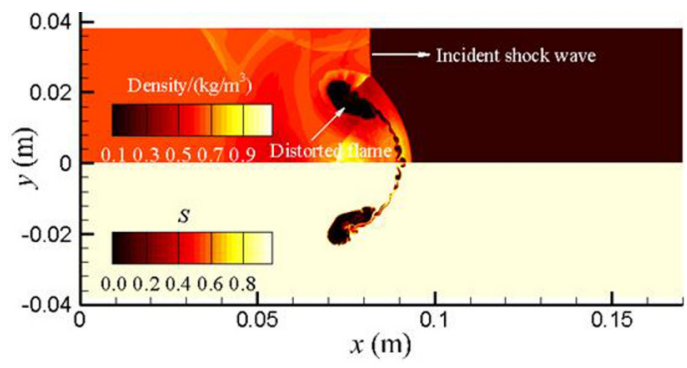

(a)

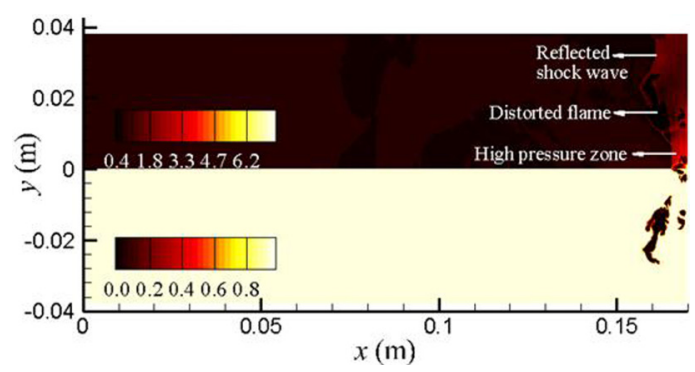

(c)

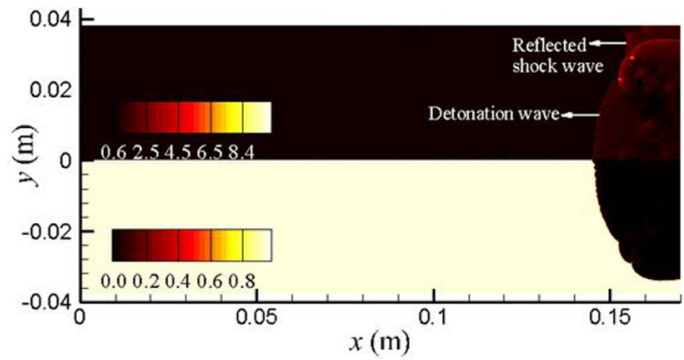

(e)

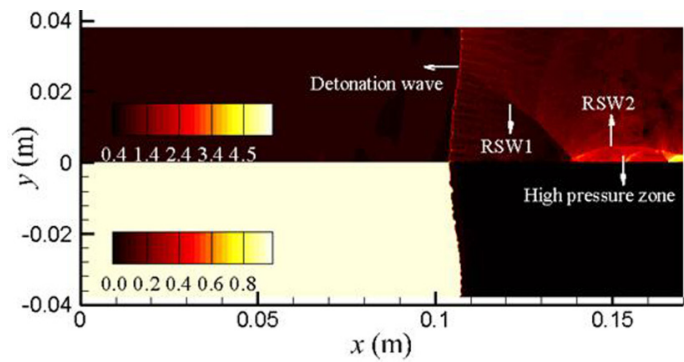

(g)

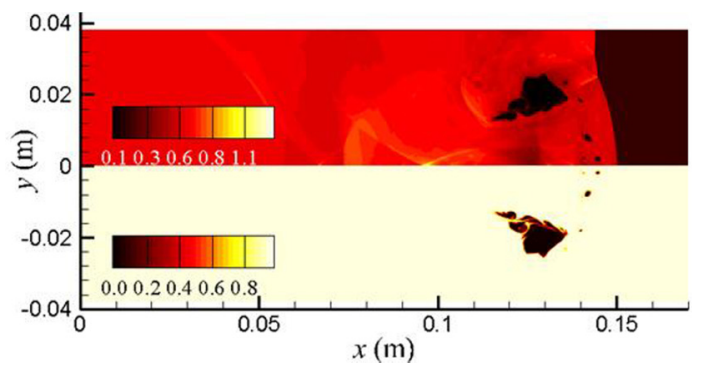

(b)

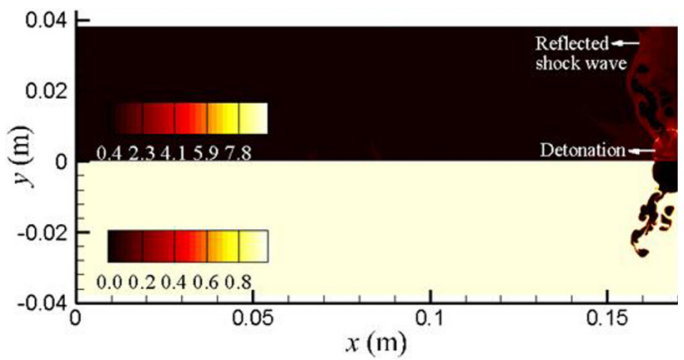

(d)

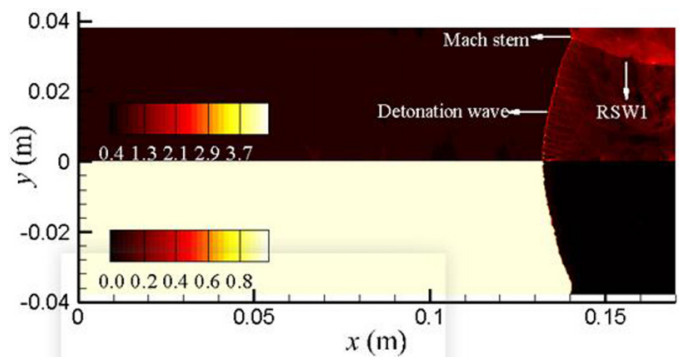

(f)

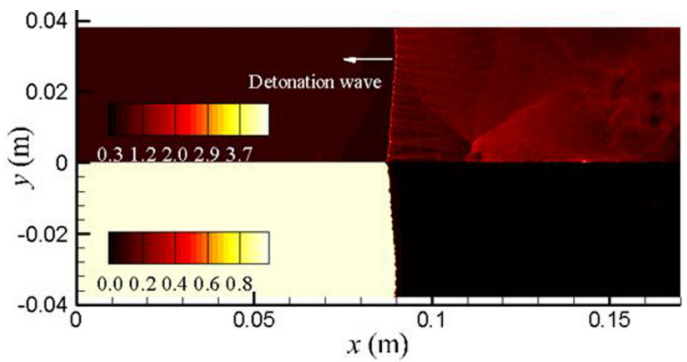

(h)

FIG. 4. Computational density and reactive gas mixture mass images (Case 1) (a) $t=75.7 \mu \mathrm{s}$, (b) $t=148.7 \mu \mathrm{s}$, (c) $t=204.7 \mu \mathrm{s}$, (d) $t=210.3 \mu \mathrm{s}$, (e) $t=222.0 \mu \mathrm{s}$, (f) $t=231.2 \mu \mathrm{s}$, (g) $t=251.4 \mu \mathrm{s}$, and (h) $t=263.0 \mu \mathrm{s}$.

noted that a shock bifurcation emerges in the vicinity of the top wall at the same time, and a similar structure called a "strange wave" can be seen (see Refs. [3,5,6]). The reason is that the leftward reflected shock wave propagates in a reverse flow with a high transverse velocity gradient, which promotes the reflected shock to cause the reverse $\lambda$-shaped bifurcation in the vicinity of the top wall. At $t=330.4 \mu \mathrm{s}$, the original leftward reflected shock wave and the RSW2 wave are merged into a planar reflected shock wave, and the shock bifurcation is further developed. Also, the area of the distorted flame is still small at this time. However, the distorted flame expands obviously in Fig. 5(e), which indicates that the chemical reaction speed increases quickly and more fresh reactive gas mixture has been involved into the combustion process. In Fig. 5(f), not only the original distorted flame expands greatly, but also a small wall flame emerges on the top wall. Hence, a distinct increased heat release can be found during this period. Even so, there is no detonation in this inhomogeneous case. By checking the numerical result, the propagation velocity of the reflected shock wave is about $1020 \mathrm{~m} / \mathrm{s}$ and the pressure behind the reflected shock wave is about $0.55 \mathrm{MPa}$ in this case, which are both nearly half of the values of the detonation wave in case 1 .

For case 3 with the negative reactive gas mixture gradient, Fig. 6 shows the evolution of flame and waves in the reflected shock accelerated flow. A local high pressure zone is formed in Fig. 6(a) owing to the same reason as those in Figs. 4(c) and 5(a). Then, similar upward shock wave RSW1, leftward shock wave RSW2 and the shock bifurcation can be found in Figs. 6(b)-6(d). In this case, there is also no 


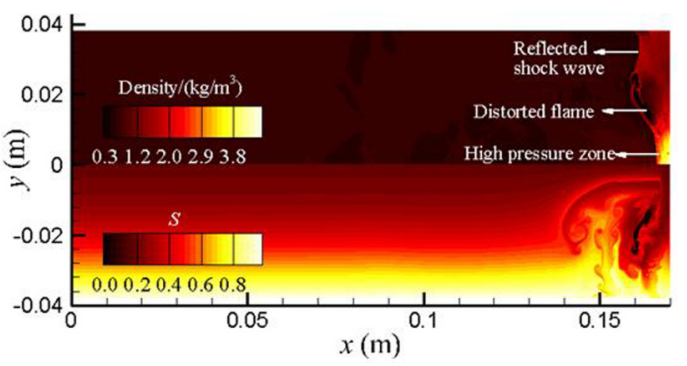

(a)

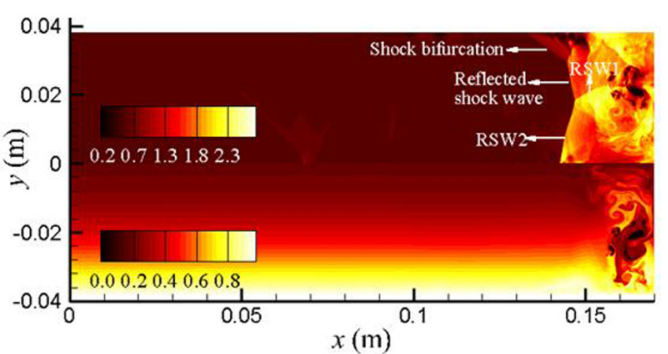

(c)

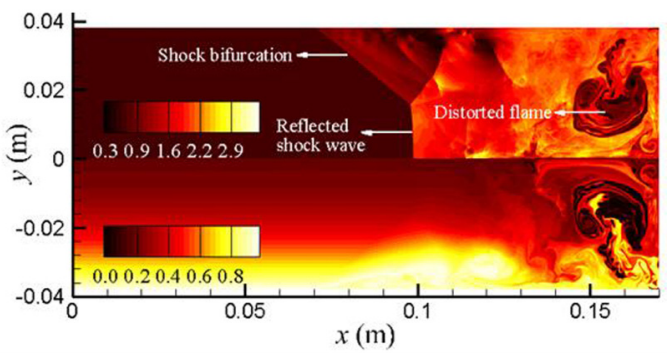

(e)

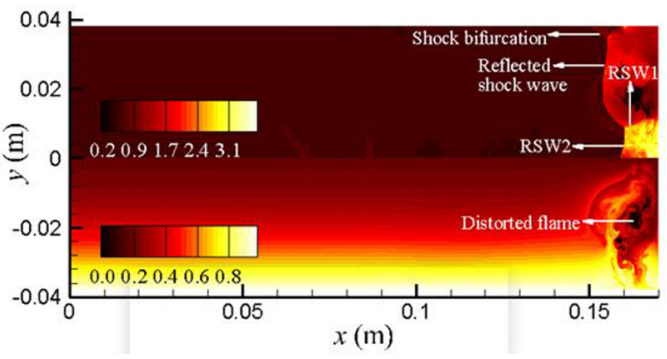

(b)

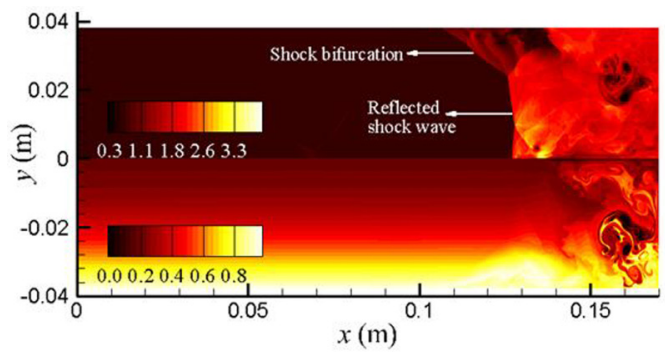

(d)

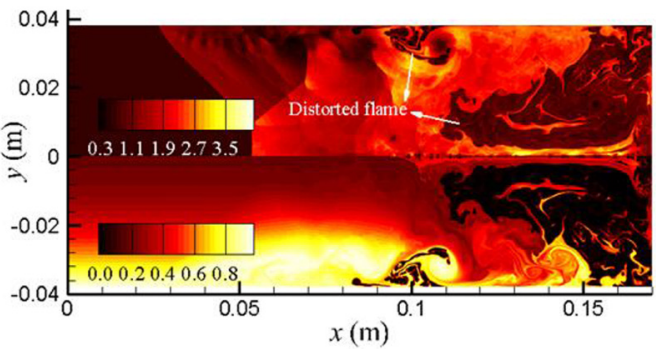

(f)

FIG. 5. Computational density and reactive gas mixture mass images (Case 2) (a) $t=204.8 \mu \mathrm{s}$, (b) $t=222.8 \mu \mathrm{s},(\mathrm{c}) t=263.0 \mu \mathrm{s}$, (d) $t=330.4 \mu \mathrm{s}$, (e) $t=410.6 \mu \mathrm{s}$, and (f) $t=529.0 \mu \mathrm{s}$.

detonation, which means the reactive gas mixture in the local high pressure zone has not been consumed thoroughly compared with the results in Figs. 4(d) and 4(e). In the meantime, the distorted flame is further compressed by the reflected shock wave, and the area of distorted flame and neighboring low density zone decreases gradually. It implies that the chemical reaction speed is relatively slow in this case, and more and more fresh reactive gas mixture with high density has been entrained into the distorted flame zone. As time goes by, the original leftward reflected shock wave and the RSW2 wave are merged into a planar reflected shock wave, and the shock bifurcation is further developed in Figs. 6(e) and 6(f). In this case, the propagation velocity of the reflected shock wave is about $885 \mathrm{~m} / \mathrm{s}$ and the pressure behind the reflected shock wave is about $0.44 \mathrm{MPa}$. Hence, overall, it is easy to find that the combustion intensity of case 3 is the weakest among the three different cases, and the inhomogeneity of fresh reactive gas mixture cannot promote the detonation initiation in this study.

\section{Integral diagnostics}

In order to deepen the understanding of the evolution of flames with time, the quantitative analyses are applied to the flame evolution by using the integral diagnostics. The flame volume $V$ is first defined as follows:

$$
V=\int_{F} d V,
$$

where the subscript $F$ represents the high temperature flame zone (defined as $S \leqslant 0.99$ and $T>1000 \mathrm{~K}$ ).

Figure 7 presents the evolution of the dimensionless flame volume $V / V_{0}$ with time ( $V_{0}$ is the initial flame volume). The twice compression stages due to the impingement of the incident and reflected shock waves, respectively, are distinctively displayed for both cases 1 and 2, wherein the values of flame volume decrease. Besides, the twice expansion stages behind the incident and reflected shock, respectively, are also distinctively displayed both for the two cases. From Fig. 7, it is easy to find that the distorted flame volume in the homogenous case is slightly larger than that in case 2 behind the incident shock wave, which implies that the combustion process in the inhomogeneous case is inhibited to a certain extent compared with that in case 1 . Moreover, the discrepancy of the flame volume in these two cases becomes more obvious behind the reflected shock wave $(t>204 \mu \mathrm{s})$. The increasing flame volume indicates that the combustion processes in both cases are enhanced owing to the reflected shock impingement. Due 


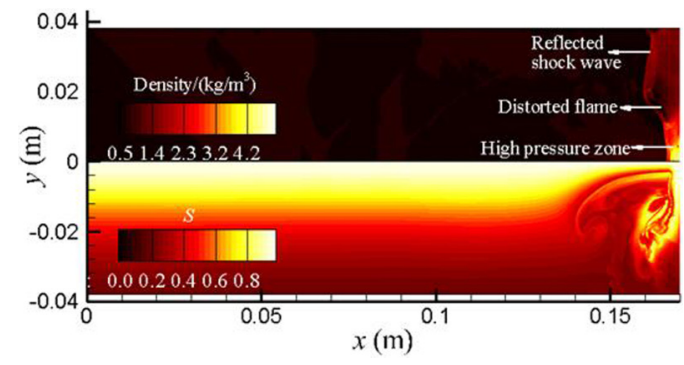

(a)

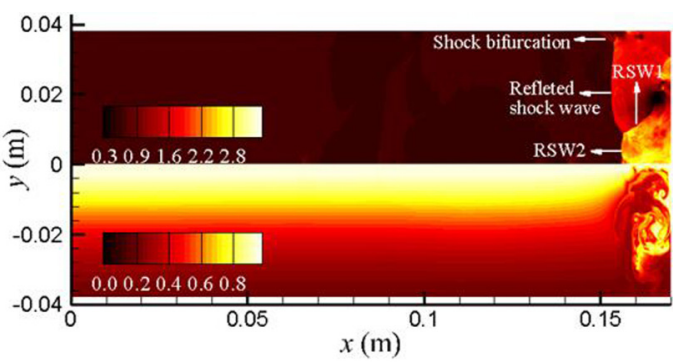

(c)

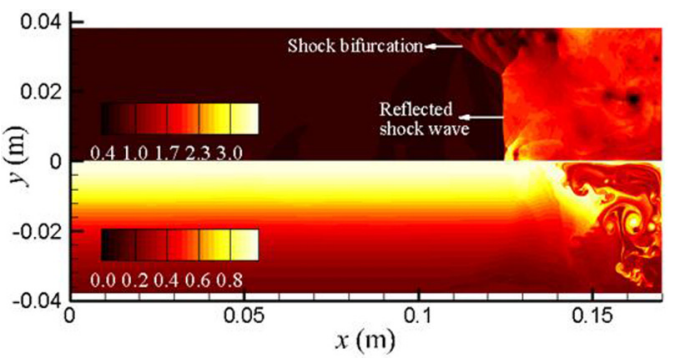

(e)

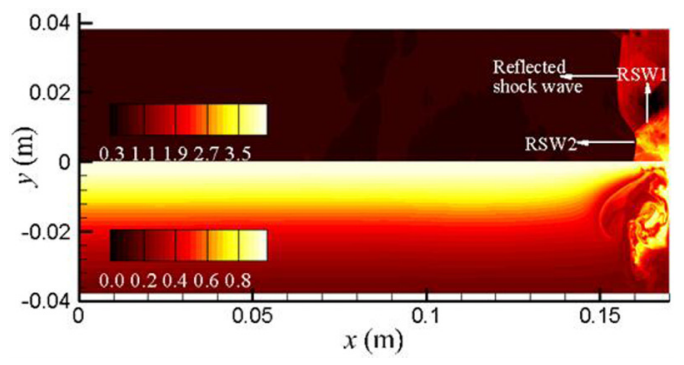

(b)

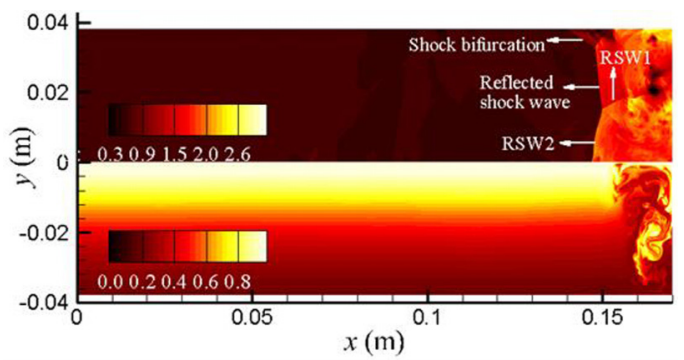

(d)

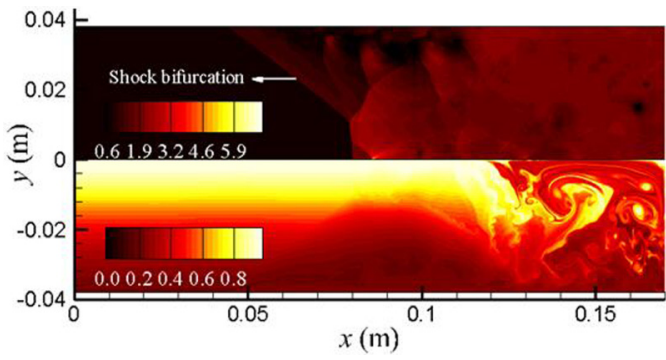

(f)

FIG. 6. Computational density and reactive gas mixture mass images (Case 3) (a) $t=205.8 \mu \mathrm{s}$, (b) $t=229.9 \mu \mathrm{s}$, (c) $t=231.4 \mu \mathrm{s}$, (d) $t=252.1 \mu \mathrm{s}$, (e) $t=340.5 \mu \mathrm{s}$, and (f) $t=480.9 \mu \mathrm{s}$.

to a detonation emerging in case 1 (as shown in Fig. 4), the flame volume increases drastically compared with that in case 2 . On the other hand, for case 3 with the negative reactive gas mixture gradient, the flame volume decreases with time from the beginning to the end, and the distorted high temperature flame zone vanishes at $t=300 \mu \mathrm{s}$. This variation of flame volume implies the combustion process in case 3 is the weakest among the three different cases, and the released

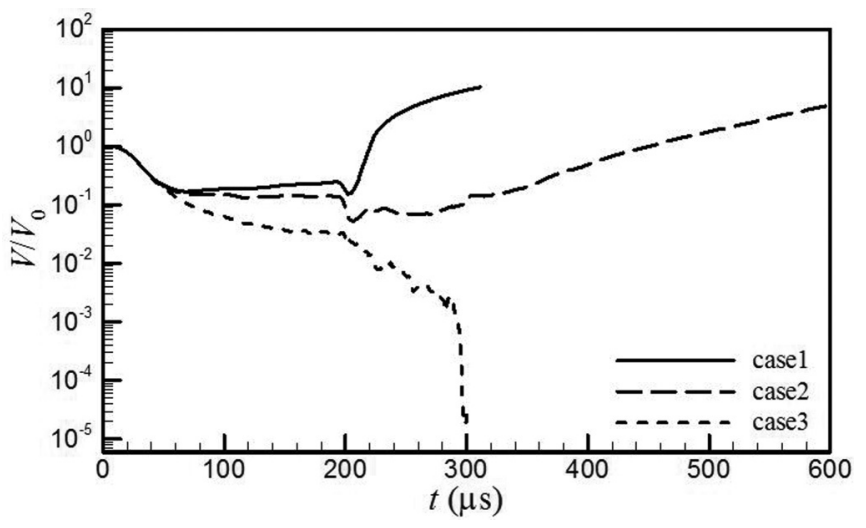

FIG. 7. Evolution of the flame volume. heat owing to combustion is less than the heat loss from the high flame zone to the surrounding fresh unburned reactive gas.

An important aspect of shock-flame interaction is the mixing between the unburned fresh reactive gas mixture and the flame. In this study, a dimensionless quantity $\xi$ is utilized to represent the ratio of unburned to burned regions in the flame zone, which can be regarded as the mixing ratio between the unburned fresh reactive gas mixture and the flame to an extent:

$$
\xi=\int_{F} X d V / \int_{F} d V,
$$

where $X$ is the unburned fresh reactive gas mixture volume fraction. Thus, it can be regarded that the mixing process dominates the flame evolution when the value of $\xi$ increases, while the combustion process gradually dominates the flame evolution when the value of $\xi$ decreases. The evolution of the ratio of unburned to burned regions in the flame zone for the three different cases is plotted in Fig. 8. It is obvious that the value of $\xi$ rises rapidly with time in case 3, thus the mixing process dominates the flame evolution all the time. Furthermore, the value of $\xi$ approaches to unity after $220 \mu \mathrm{s}$, which indicates that there is basically no combustion at this time. Before the reflected shock wave impinges on the distorted flame, the value of $\xi$ in the homogeneous case is higher than 


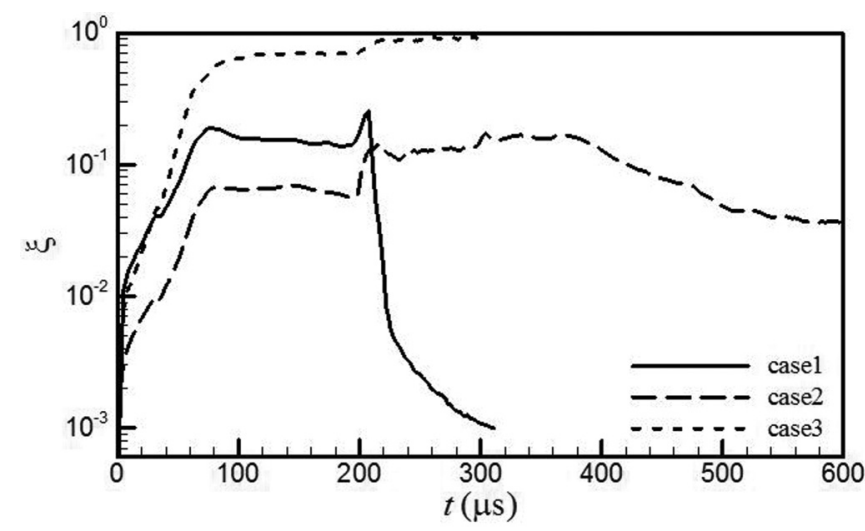

FIG. 8. Evolution of the ratio of unburned to burned regions in the flame zone.

that in case 2 although the combustion intensity in case 1 is stronger. The main reason is that the unburned fresh reactive gas surrounding the high temperature flame in the inhomogeneous case is leaner than that in the homogeneous case, which leads to a small mixing ratio between the unburned fresh reactive gas mixture and the flame in case 2. However, the flow fields alter tremendously behind the reflected shock wave owing to the emerging detonation in case 1 . Hence, the greatly enhanced combustion intensity in the homogeneous case induces the value of $\xi$ decreases sharply compared with the other two inhomogeneous cases.

Figure 9 shows the total heat release rate $d h / d t$, as a function of time for all of the three different cases, which can be expressed as

$$
\frac{d h}{d t}=\int_{F} \rho \dot{\omega} q \mathrm{~d} V .
$$

From this figure, it can be found that the values of the total heat release rate all increase when the incident and reflected shock waves impinge on the flame. The change trend suggests the flame distortions could greatly extend the contact area of the flame with the ambient unburned fresh reactive gas mixture, promote the mixing between the unburned/burned gases, and therefore enhance the chemical heat release. For the homogeneous case, the total heat release rate remains unchanged basically behind the incident and reflected shock

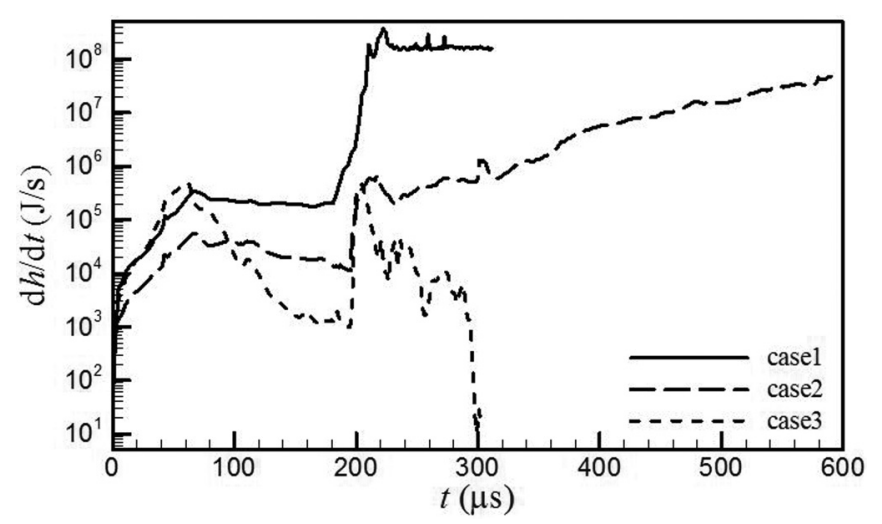

FIG. 9. Evolution of the total heat release rate of flame. waves, and it is also the highest value among the three cases. Especially for the period behind the reflected shock wave, a jump of two orders of magnitude of the total heat release rate can be found, which is in accordance with the emergence of detonation in Fig. 4. For case 2 with the positive reactive gas mixture gradient, the total heat release rate increases continuously behind the reflected shock wave, and this also corresponds to the expanding flame zone in Figs. 5 and 7. However, compared with the total heat release rate in cases 1 and 2 , the heat release rate decreases behind the incident and reflected shock waves in case 3 , so the flame volume decreases in Fig. 7. Therefore, it is concluded that the combustion intensity with the homogeneous reactive gas mixture (case 1) is the strongest, the combustion intensity with the positive reactive gas mixture gradient (case 2) is medium, and the combustion intensity with the negative reactive gas mixture gradient (case 3) is the weakest. Moreover, the impingement of reflected shock wave with the distorted flame could greatly promote the combustion process, enhance the heat release, and affect the combustion characteristics of the flow fields.

In fact, the vorticity generation of the flame zone induced by shock waves impingement can enhance the mixing between the ambient unburned fresh reactive gas and the flame, thus promoting the combustion process and increasing the heat release rate. Combustion, however, can either inhibit or promote the vorticity generation. Specifically speaking, the enhanced combustion process could consume more fresh reactive gas that is entrained by vorticities, and thus the flame expands quickly and the surface of the flame becomes smooth, so the vorticity generation is weakened to some extent. Meanwhile, the enhanced combustion process could greatly alter the flow fields characteristics, thus the velocity gradient, pressure gradient, and density gradient will be intensified. Consequently, the vorticity generation is promoted in this way. To further clarify the intrinsic mechanism of vorticity generation with time in the flame zone, the vorticity kinetics equation of the two-dimensional compressible fluid is given as

$$
\begin{aligned}
\frac{D \omega}{D t}= & -\omega\left(\frac{\partial u}{\partial x}+\frac{\partial v}{\partial y}\right)+\frac{1}{\rho^{2}}\left(\frac{\partial \rho}{\partial x} \frac{\partial p}{\partial y}-\frac{\partial \rho}{\partial y} \frac{\partial p}{\partial x}\right) \\
& +v\left(\frac{\partial^{2} \omega}{\partial x^{2}}+\frac{\partial^{2} \omega}{\partial y^{2}}\right),
\end{aligned}
$$

where the first and second transport terms in the right-hand of Eq. (7) are the compression term $\omega_{\mathrm{c}}$ and baroclinic term $\omega_{\mathrm{b}}$, respectively. The third term represents the viscosity term $\omega_{\mathrm{d}}$. Figure 10 illustrates the evolution of the sum of the absolute values for the different transport terms within the flame zone, which are denoted by $\sum\left|\omega_{c}\right|, \sum\left|\omega_{b}\right|$, and $\sum\left|\omega_{d}\right|$, respectively. Therefore, a higher value of the transport term indicates that this transport term has a greater influence on the vorticity generation. From Fig. 10, it is easy to find that the absolute value of each transport term decreases with the weaken of the combustion intensity (from case 1 to case 3 ), and the value of $\sum\left|\omega_{d}\right|$ is almost the smallest among the three cases, which implies that the influence of the viscosity term on the vorticity generation can be negligible compared with the other two transport terms. Also, a noteworthy feature of Fig. 10 is that, before the reflected shock wave, the value of 


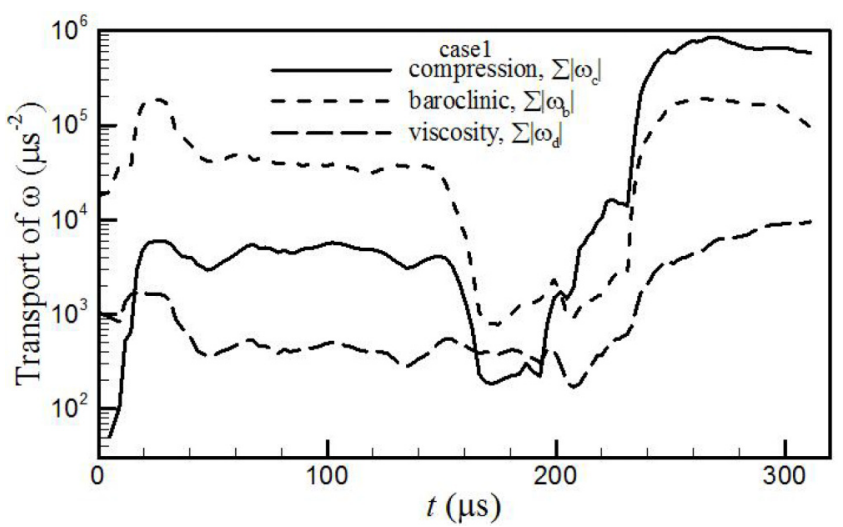

(a)

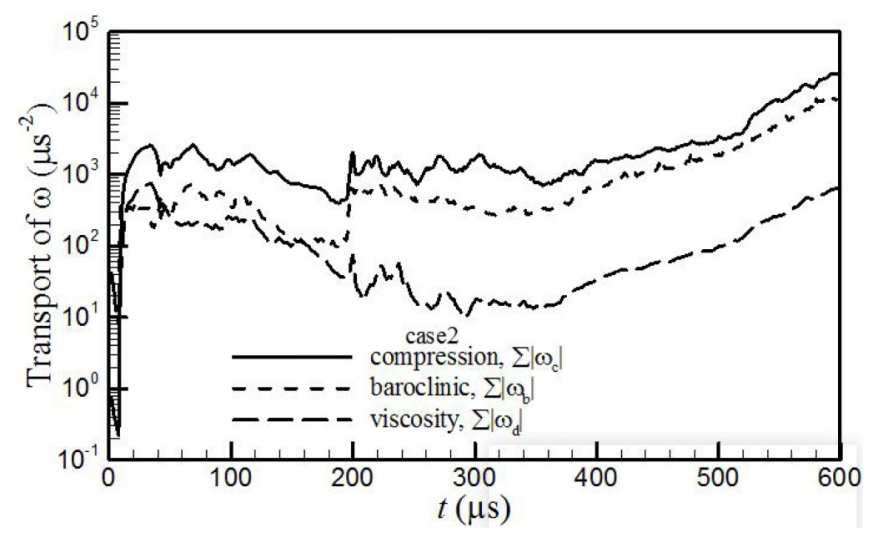

(b)

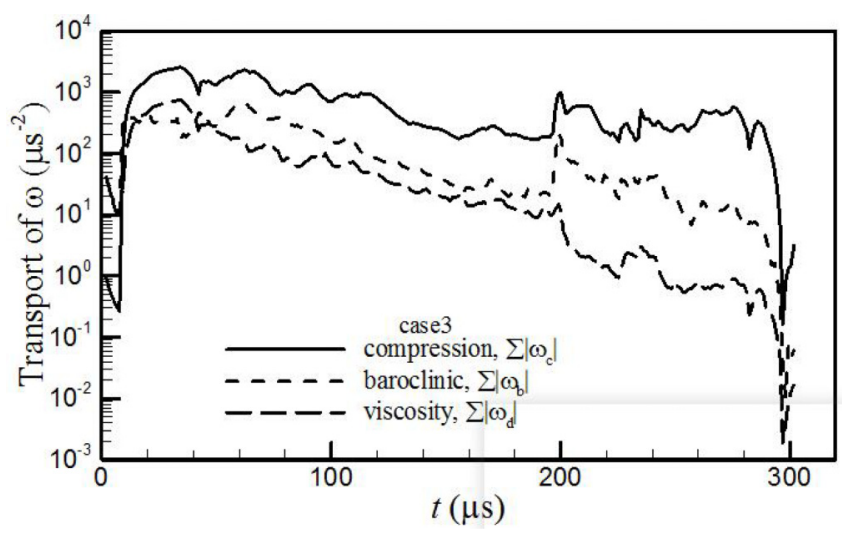

(c)

FIG. 10. Evolutions of the different transport terms for different cases (a) case 1 , (b) case 2 , and (c) case 3 .

$\sum\left|\omega_{b}\right|$ is the largest among the three transport terms in case 1 , which means the baroclinic effect (the misalignment between the pressure and density gradients) dominates the vorticity generation of the flame zone during this period. Whereas for cases 2 and 3, the values of $\sum\left|\omega_{c}\right|$ are both the largest values, so the compression effect dominates in the vorticity generation in these two cases. After the interaction between the reflected shock wave and the distorted flame, the absolute value of each transport term increases in cases 1 and 2 owing to the enhanced combustion intensity. Especially for the homogeneous case, the value of $\sum\left|\omega_{c}\right|$ increases more quickly than the value of $\sum\left|\omega_{b}\right|$. Thus, it indicates the influence of compression effect is stronger than that of the baroclinic effect during this period, which can be attributed to the influence of the emerging detonation. But for case 3, all the transport terms decrease behind the incident and reflected shock waves owing to the weakest combustion intensity. The different influences of the transport terms on the vorticity generation reveal the influence of different reactive gas mixture gradients on the shock-flame interaction process clearly.

\section{CONCLUSIONS}

In this study, the spherical flame instability and acceleration induced by incident shock and its reflected waves are numerically studied for an ethylene-oxygen-nitrogen gas mixture, with the same high initial shock Mach number $(\mathrm{Ma}=$ 2.51). In particular, the influences of different initial reactive gas mixture gradients on the shock-flame interaction are investigated. The main conclusions are summarized as follows:

(1) The combustion intensities of case 1 , case 2, and case 3 correspond to strong, medium, and weak combustion, respectively. Hence, a detonation emerges in the homogenous reactive gas mixture case, which can consume the fresh reactive gas mixture rapidly. Whereas for the inhomogeneous cases, no detonation emerges, but different flame evolution is observed according to the positive or negative reactive gas mixture gradients. Besides, a distinct shock bifurcation emerges from the vicinity of the top wall owing to the leftward reflected shock wave propagating in a reverse flow with a high transverse velocity gradient in the inhomogeneous cases.

(2) The flame volume and heat release rate both increase in cases 1 and 2 but decrease in case 3 , especially for the period behind the reflected shock wave. To the contrary, the ratio of unburned to burned regions in the flame zone decreases in cases 1 and 2 but increases in case 3 .

(3) Before the reflected shock wave impinges on the distorted flame, the baroclinic effect dominates the vorticity generation in the flame zone in the homogeneous case. Thereafter, the compression term dominates due to the onset of a detonation wave. However, in the inhomogeneous cases, the compression term, the baroclinic term, and the viscosity term have strong, medium, and weak influences, respectively, on the vorticity generation throughout the processes.

\section{ACKNOWLEDGMENTS}

This work was supported by the National Natural Science Foundation of China (Grants No. 11872193, No. 11402102, and No. 91641113), the Natural Science Foundation of Jiangsu Province of China (Grant No. BK20140524), the Jiangsu Overseas Visiting Scholar Program for University Prominent Young \& Middle-aged Teachers and Presidents, and the Youth Talent Cultivation Plan of Jiangsu University. 
[1] J. Yang, T. Kubota, and E. E. Zukoski, AIAA J. 31, 854 (1994).

[2] H. H. Teng, Z. L. Jiang, and H. D. Ng, J. Fluid Mech. 744, 111 (2014).

[3] E. S. Oran and V. N. Gamezo, Combust. Flame. 148, 4 (2007).

[4] G. O. Thomas, R. Bambrey, and C. Brown, Combust. Theory Model. 5, 573 (2001).

[5] E. S. Oran, V. N. Gamezo, and A. M. Khokhlov, 40th AIAA Aerospace Sciences Meeting \& Exhibit, Paper 2002-0776, 14-20 January 2002 (AIAA, Reston, VA, USA, 2002).

[6] V. N. Gamezo, E. S. Oran, and A. M. Khokhlov, Proc. Combust. Inst. 30, 1841 (2005).

[7] H. H. Teng, Z. L. Jiang, and Z. M. Hu, Acta Mech. Sin. 23, 343 (2007).

[8] B. D. Taylor, R. W. Houim, D. A. Kessler, V. N. Gamezo, and E. S. Oran, 51st AIAA Aerospace Sciences Meeting including the New Horizons Forum and Aerospace Exposition, Paper 2013-1171, 7-13 January 2013, Grapevine (AIAA, Texas, USA, 2013).

[9] Y. J. Zhu, G. Dong, and Y. X. Liu, Combust. Sci. Technol. 185, 1415 (2013).
[10] Y. J. Zhu, G. Dong, Y. X. Liu, B. C. Fan, and H. Jiang, Acta Mech. Sin. 29, 54 (2013).

[11] L. R. Boeck, J. Hasslberger, and T. Sattelmayer, Combust. Sci. Technol. 186, 1650 (2014).

[12] L. R. Boeck, F. M. Berger, J. Hasslberger, and T. Sattelmayer, Shock Waves 26, 181 (2016).

[13] C. J. Wang and J. X. Wen, Int. J. Hydrogen Energy 42, 7657 (2017).

[14] C. Huete, T. Jin, D. Martínez-Ruiz, and K. Luo, Phys. Rev. E 96, 053104 (2017).

[15] G. S. Jiang and C. W. Shu, J. Comput. Phys. 126, 202 (1996).

[16] Y. J. Zhu, L. Yu, J. F. Pan, Z. H. Pan, and P. G. Zhang, Phys. Fluids 29, 126105 (2017).

[17] Y. J. Zhu, Z. W. Yang, Z. H. Pan, P. G. Zhang, and J. F. Pan, Comput. Fluids 177, 78 (2018).

[18] H. Jiang, G. Dong, X. Chen, and B. Li, Combust. Flame. 169, 229 (2016).

[19] G. Dong, B. Fan, and J. Ye, Shock Waves 17, 409 (2008).

[20] B. J. Mcbride and S. Gordon, National Aeronautics and Space Administration Lewis Research Center, NASA, Technical Report No. RP-1311-P2, Cleveland, Ohio, 1996. 